\title{
An apparatus for measuring nonlinear viscoelasticity of minerals at high temperature
}

\author{
Ri Cao ${ }^{1}$, Lars N. Hansen ${ }^{2}$, Christopher A. Thom ${ }^{1}$, David Wallis ${ }^{3}$ \\ ${ }^{1}$ Department of Earth Sciences, University of Oxford, Oxford, OX1 3AN, United Kingdom \\ ${ }^{2}$ Department of Earth and Environmental Sciences, University of Minnesota, Minneapolis, \\ 55455, United States of America \\ ${ }^{3}$ Department of Earth Sciences, University of Cambridge, Cambridge, CB2 3EQ, United \\ Kingdom
}

\begin{abstract}
We describe a high-temperature, uniaxial creep apparatus designed to investigate nonlinear attenuation of materials over a wide range of temperatures $\left(25-1300^{\circ} \mathrm{C}\right)$ using forced oscillations combined with a bias stress. This apparatus is primarily designed for investigation of minerals and rocks with high melting temperatures. An oscillatory compressional stress is used to determine attenuation and Young's modulus at frequencies of $10^{-2}$ to $10^{2} \mathrm{~Hz}$ and high stress amplitudes (>0.1 MPa). Large bias stresses are applied in addition to the oscillatory stresses such that attenuation tests are conducted simultaneously with ongoing creep.
\end{abstract}

The complex compliance of the apparatus was characterized by conducting calibration tests on orientated crystals of sapphire. The real part of the apparatus compliance exhibits a dependence on sample length and frequency, whereas the imaginary part is only dependent on frequency. The complex compliance is not dependent on the oscillation amplitude or the bias stress. We assess the accuracy and precision of this calibration by comparing measurements of the attenuation and Young's modulus of stainless steel, aluminum, and acrylic to previously published values. We outline a set of criteria defining the conditions over which this apparatus can accurately determine the attenuation and Young's modulus of a sample based on the sample length and expected values of attenuation and Young's modulus.

Keywords: viscoelasticity, transient creep, amplitude-dependent attenuation, apparatus compliance 


\section{Introduction}

Viscoelasticity describes the mechanical properties of materials that exhibit both viscous and elastic behavior, with the relative proportion of the two depending on the timescale of observation (Lakes, 2009). Rocks clearly exhibit viscoelastic behavior since they act elastically on very short timescales and viscously on very long timescales. Most previous investigations of viscoelasticity in geological materials have been focused on seismological properties such as seismic-wave attenuation (Abers et al., 2014; Cline et al., 2018; Farla et al., 2012; Jackson and Faul, 2010; Lau and Holtzman, 2019; McCarthy et al., 2011; Takei et al., 2011) and therefore have been conducted at low stress amplitudes $\left(<10^{-5}\right.$ in elastic strain) in the linear regime. However, increasingly complex viscoelastic phenomena may occur as stress amplitudes are increased and the behavior becomes nonlinear. Nonlinear viscoelasticity likely characterizes the response of the solid earth in situations with abrupt stress changes, such as post-seismic relaxation (Agata et al., 2019; Freed et al., 2012; Masuti et al., 2016; Moore et al., 2017; Qiu et al., 2018).

Unfortunately, currently available apparatus have a variety of limitations for investigating nonlinear viscoelasticity in geological materials. Typical viscoelastic measurements of geological materials have been performed by applying a periodic stress that oscillates between a positive stress and a negative stress, that is, the time-averaged stress or bias stress is zero. These measurements include experiments conducted with forced oscillations (Gribb and Cooper, 1998; Jackson and Paterson, 1993; Li and Weidner, 2007; Sundberg and Cooper, 2010) or in a resonant torsion pendulum (Gueguen et al., 1989). However, nonlinear viscoelasticity in crystalline materials tends to arise from the dynamics of lattice dislocations. The dislocation density and any internal stress fields scale with the quasi-static applied stress (Bai and Kohlstedt, 1992; Twiss, 1986), and therefore, if the time-averaged stress is negligible, then there is a tendency for initial dislocation densities and internal stresses to relax. Some researchers have predeformed samples under a quasi-static stress in a separate apparatus to induce high dislocation densities prior to attenuation tests at elevated temperatures (Farla et al., 2012). Temperatures in these tests are kept at moderate levels to prevent dislocation recovery, but although dislocation densities may not change dramatically during the subsequent attenuation test, the internal stresses still likely relax substantially and rapidly due to modest rearrangements of the dislocations (Wallis et al., 2020). 
Application of a non-zero bias stress during oscillation tests overcomes some of these difficulties. A quasi-static bias stress maintains dislocation densities and internal stress levels throughout superimposed oscillation tests (e.g., Sapozhnikov and Kustov, 1997). Application of very large quasi-static bias stresses such that the material is actively creeping during simultaneous oscillations also allows dislocation dynamics to be investigated through features such as the acoustoelastic effect (e.g., Kaiser and Pechhold, 1969). Furthermore, Gremaud et al. (1987) demonstrated that superimposing low-amplitude forced oscillations on top of largeamplitude, low-frequency oscillations of a bias stress can allow one to uniquely identify the aspects of the dislocation dynamics contributing to energy dissipation. Thus, future investigations of nonlinear viscoelasticity in geological materials will greatly benefit from being able to apply a controlled, non-zero bias stress during attenuation measurements. We note that several existing apparatus (McCarthy and Cooper, 2016; Takei et al., 2011) have been designed for geological applications that can apply a controlled bias stress during oscillatory experiments. However, they are limited to low temperatures typically used to investigate geological materials with very low melting temperatures (e.g., ice) or organic compounds that are reasonable analogs for geological materials (e.g., borneol).

Here we introduce a novel apparatus specifically designed to overcome the limitations of existing apparatus. This new apparatus is based on traditional, high-temperature, uniaxial deadload rigs designed for creep tests under controlled environments. The key modification of traditional designs is the addition of a piezoelectric actuator for applying oscillating loads. This actuator allows us to apply a controlled bias stress while simultaneously applying a superimposed oscillatory stress. Here we describe the main features of the apparatus and outline a series of calibration experiments to define the limitations in material properties that can be explored.

\section{Apparatus}

\subsection{Loading frame and actuation}

Figure 1 depicts the key components of the uniaxial deformation apparatus. The primary loading frame consists of two steel sheets with cutouts for access points. An upper crosshead is fixed in place and acts as a mounting point for a water-cooled bracket that holds the upper $\mathrm{SiC}$ piston. The bottom $\mathrm{SiC}$ piston is mounted in series with a piezoelectric actuator and a load cell. The load cell sits atop a steel piston with a flange such that the piston can be supported in one of two ways. First, as depicted in Figure 1b, a collar that is driven by a worm gear mounted 
on the lower crosshead can transfer the load from the loading column to the lower crosshead and frame if the collar is high enough to contact the flange on the steel piston. Alternatively, a central piston that goes through the collar can be loaded from below by the lower electromechanical actuator. This configuration transfers the load to the electromechanical actuator and its mounting points at the base of the main loading frame.

a

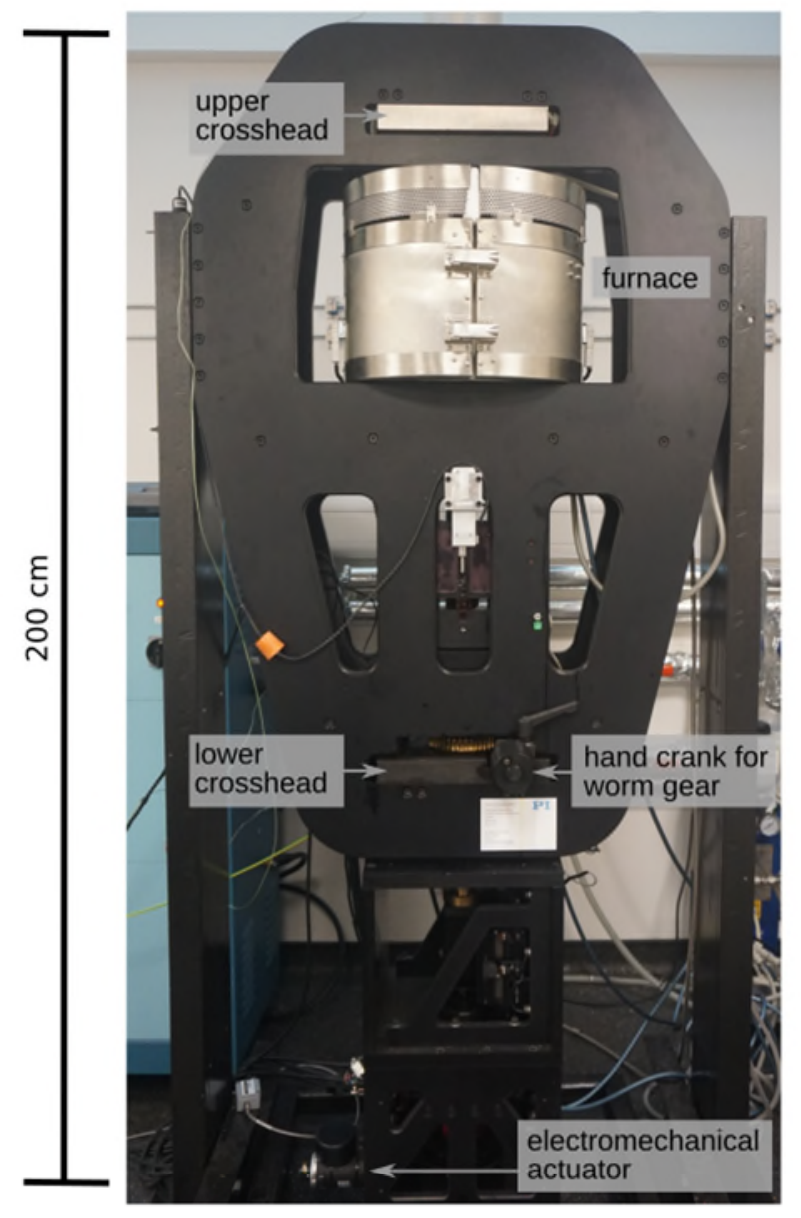

b

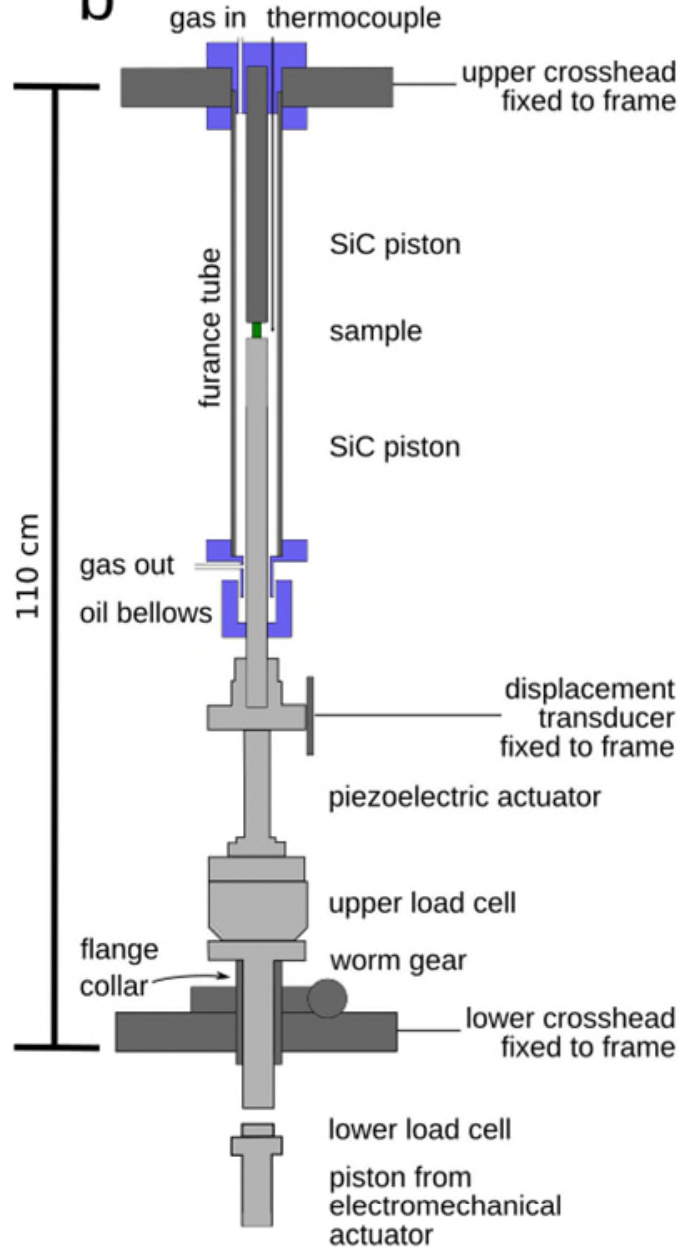

Figure 1: High-temperature uniaxial creep apparatus for investigation of viscoelastic properties. (a) Photograph of the apparatus in the Rock Rheology Lab at the University of Oxford with annotations of key visible features. (b) Schematic illustration of the primary components of the loading column. Dark-gray components are fixed to the loading frame. Blue components are water cooled. The displacement transducer (a linear encoder) measures displacement of the lightgray components relative to the dark-gray components. The electromechanical actuator is decoupled from the loading column in the pictured configuration, and the collar is fully raised to support the flanged piston, transferring load to the lower crosshead.

In the first configuration, the electromechanical actuator is effectively removed from the system, and the lower SiC piston is primarily driven by the piezoelectric actuator. The piezoelectric actuator (model P-025.80P produced by Physik Instrumente) is set up in a closed control loop with the upper load cell to enable constant loads to be maintained. A strain gauge 
is affixed to the piezoelectric actuator to monitor the available throw of the actuator (a maximum of $\sim 180 \mu \mathrm{m}$ ). If the piezoelectric actuator runs out of throw, then the collar, steel piston, load cell, and piezoelectric actuator can all be driven upwards by hand using the worm gear. Because of the fast response of the control loop, this hand adjustment can be made while a constant load is being maintained. In the second configuration, the loading column is driven by the electromechanical actuator, which has a total throw of $\sim 3 \mathrm{~cm}$. This ball-screw driven actuator is configured for actuation to be controlled by position or displacement rate.

\subsection{Displacement and load measurement}

Figure 1 also depicts the critical components for measuring sample displacement and the applied load. The primary displacement transducer is a linear encoder with the readhead mounted on the loading column just above the load cell and the encoded scale mounted on the loading frame, providing a measurement of displacement of the bottom of the loading column (and therefore the bottom $\mathrm{SiC}$ piston) relative to the loading frame. Displacement is measured at a resolution of $10 \mathrm{~nm}$. We have experimented with a transducer with $1.5 \mathrm{~nm}$ resolution and found essentially no improvement in the results presented below. A "top hat" with electrical feedthroughs (not pictured) is also affixed atop the apparatus to provide space for an extensometer to sit within the gas environment. An extensometer is valuable for long-term creep experiments since it provides temperature compensation. However, we have found the extensometer to be subject to significant resonance during forced oscillations, and therefore it is removed from the apparatus during those experiments and not described in further detail here.

The load is measured by a load cell with a range of $9 \mathrm{kN}$ and a repeatability of $1 \mathrm{~N}$. The loading frame and loading column are designed for maximum loads of $5 \mathrm{kN}$. Due to the weight of the loading column, there is a systematic background load for the apparatus of $98 \mathrm{~N}$.

\subsection{High-temperature furnace and chemical environment}

As depicted in Figure 1, the sample is heated with a gas-mixing tube furnace that encloses an alumina tube, the sample assembly, and the two SiC pistons. The top of the furnace tube is affixed to a water-cooled mounting bracket on the upper crosshead, and the bottom to a watercooled fitting with a frictionless seal for the bottom $\mathrm{SiC}$ piston to pass through. The maximum operating temperature is approximately $1330^{\circ} \mathrm{C}$ and is limited by the maximum power of the heating element of the furnace. The sample temperature is controlled within $\pm 0.1^{\circ} \mathrm{C}$ using a $\mathrm{K}$ - 
type thermocouple placed at the height of the centre of the samples, approximately $15 \mathrm{~mm}$ from the centerline of the loading column.

For future experiments on materials that are sensitive to the oxygen fugacity, a controlled ratio of $\mathrm{CO}_{2}$ and $\mathrm{CO}$ will be fluxed through the furnace tube throughout the experiment. For most of the calibration experiments described below, the samples were stable in air at the conditions of interest, and therefore modification of the chemical environment within the furnace was not necessary.

\subsection{Characteristics of calibration samples}

A variety of samples were used in the calibration tests described below. We primarily focus on single-crystal sapphire oriented for compression along the [0001] axis. Sapphire deformed in this orientation is ideal for calibrating the apparatus stiffness because of its wellcharacterized Young's modulus of 456 GPa (Cheng et al., 2016; Wang et al., 2017) and lack of viscous deformation at room temperature (Firestone and Heuer, 1976). We tested a variety of sapphire crystals with different sample dimensions ( $4 \mathrm{~mm}, 6 \mathrm{~mm}$, and $8 \mathrm{~mm}$ in length) to examine the dependence of the apparatus compliance on sample size.

The apparatus stiffness calibration experiments described below are sensitive to irregularities in the contact between interfaces in the loading column, so superglue was used to ensure good contact between the sample and the two pistons at room temperature following the method of Takei et al., (2014).

\section{Sensitivity testing and calibration}

\subsection{Timing error in data acquisition}

To improve the capability of the apparatus to measure low magnitudes of attenuation, we must ensure the relative timing of different measured signals is as precise as possible. As illustrated in Figure 2, an impulse generated by the piezoelectric actuator results in a near-instantaneous increase in both the position and the load. However, the recorded increase in position occurs slightly ahead of the recorded increase in load, which is an erroneous feature of our data acquisition system. To quantify this timing error, we conducted several tests consisting of a rapid 10-N increase in load and estimated the timing offset by cross-correlating the position and load signals. The measured timing error is approximately $200 \mu$ s, which is independent of 
the sampling rate used for data acquisition. All data sets discussed below have been corrected for this timing error by shifting the position signal forward by $200 \mu \mathrm{s}$.

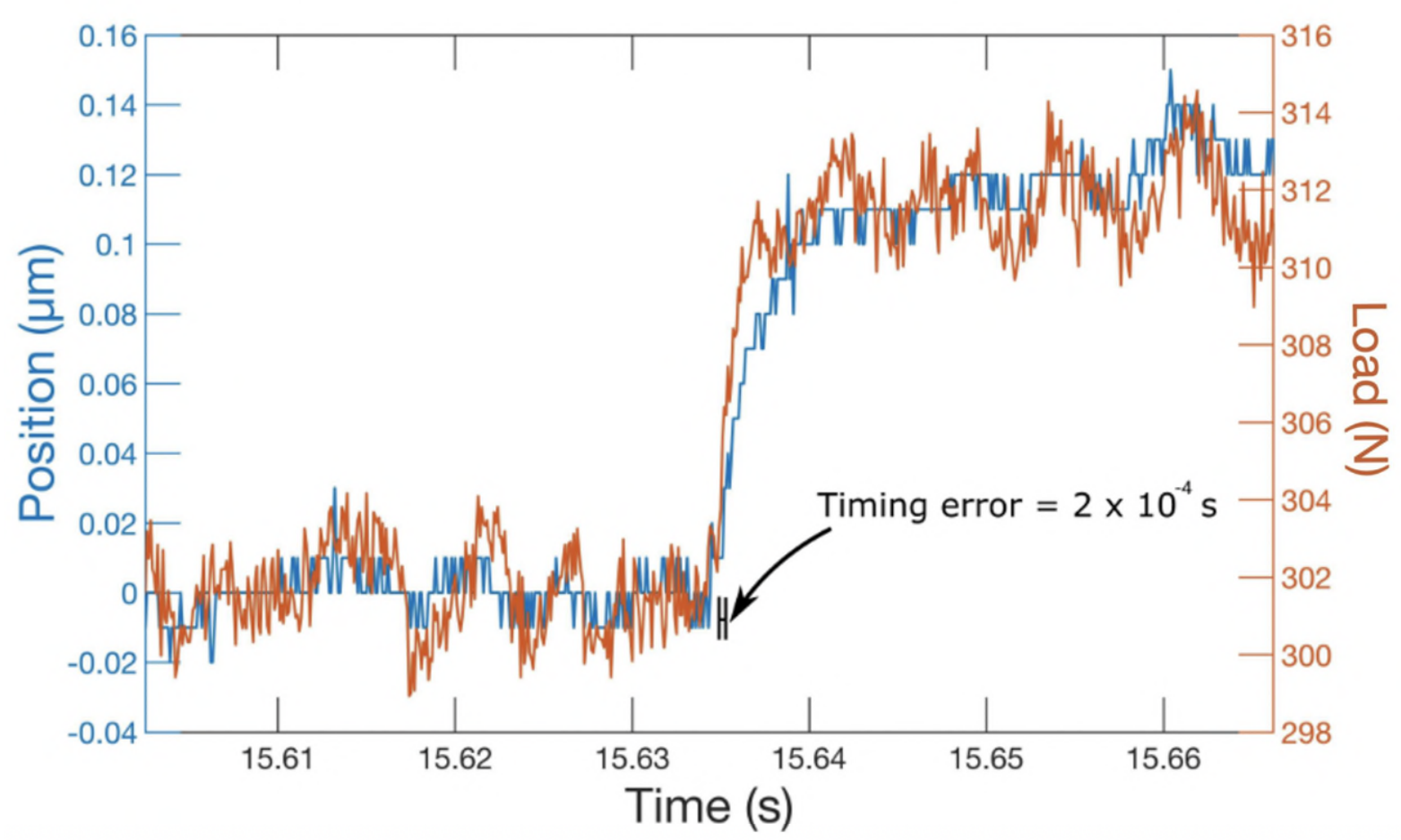

Figure 2: Rapid load increase to measure the timing error between the position and load signals. The timing error estimated by cross-correlation is $2 \times 10^{-4} \mathrm{~s}$. These data were collected with a sampling rate of $10 \mathrm{kHz}$.

\subsection{Resonant frequency}

A factor that may add an additional complication to interpretation of attenuation experiments is resonance of the apparatus. We conducted several tests on single-crystal sapphire to estimate the resonant frequency of the loading frame and loading column, as illustrated in Figure 3. 

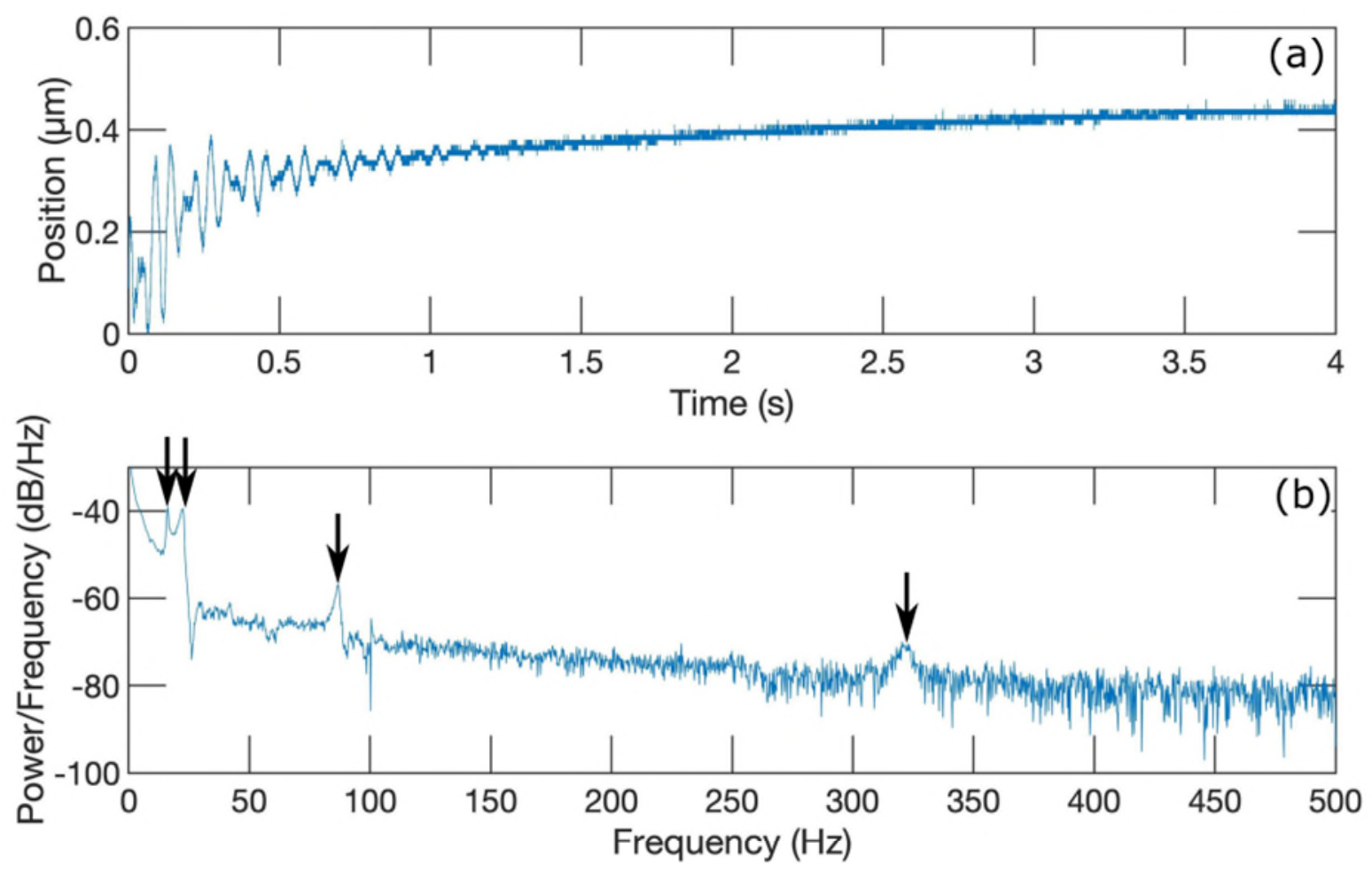

Figure 3: A rapid load increase (from $1 \mathrm{kN}$ to $4 \mathrm{kN}$ ) to evaluate the resonance of the apparatus. (a) Displacement measured as a function of time at room temperature just after the load increases. (b) Power spectrum of the time series presented in (a). The arrows indicate apparent resonant frequencies $(16 \mathrm{~Hz}, 20 \mathrm{~Hz}, 90 \mathrm{~Hz}$, and $320 \mathrm{~Hz})$.

To conduct these tests, we applied an impulse directly to the loading frame by increasing the load instantaneously from $1 \mathrm{kN}$ to $4 \mathrm{kN}$. The recorded displacement and its power spectrum are presented in Figure 3. These results indicate that the fundamental resonance occurs at a frequency of $16 \mathrm{~Hz}$, with additional peaks at approximately $20 \mathrm{~Hz}, 90 \mathrm{~Hz}$, and $320 \mathrm{~Hz}$. Therefore, we explicitly avoid these frequencies when conducting forced oscillation tests to measure the complex compliance of the apparatus.

\subsection{Methods for calculating attenuation}

An additional consideration in conducting attenuation experiments is the method by which the data are processed. We evaluated three different methods to calculate attenuation, $Q^{-1}$. A common method for calculation of attenuation (e.g., Jackson and Faul, 2010; McCarthy and Cooper, 2016; Takei et al., 2011) is from the phase shift between the stress and strain signals, 


$$
\begin{gathered}
\delta=2(\Delta t) f, \\
Q^{-1}=\tan (\delta),
\end{gathered}
$$

where $\delta$ and $\Delta t$ are the phase shift and time lag between the stress and strain signals, respectively, and $f$ is the oscillation frequency. The apparent modulus can also be calculated by

$$
E=\frac{\sigma_{0}}{\varepsilon_{0}}
$$

where $\sigma_{0}$ and $\varepsilon_{0}$ are the maximum stress and maximum strain, respectively. However, two additional methods can be used to estimate the attenuation from the same data set using the geometry of an elliptical Lissajous loop (Figure 4; Lakes, 2009, p. 60-62).

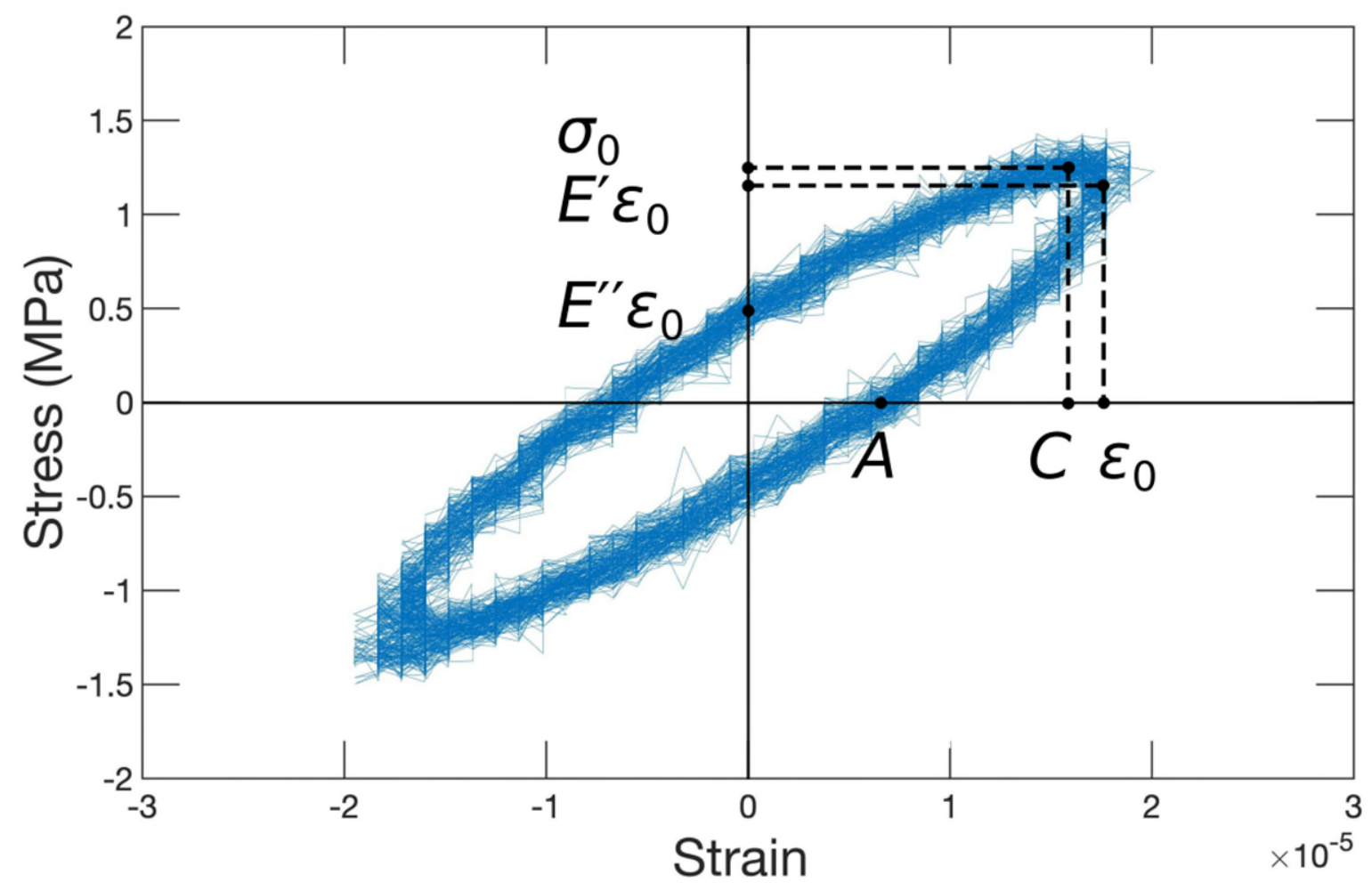

Figure 4: A typical example of a stress-strain curve from a forced-oscillation measurement on single-crystal sapphire. These data have not been corrected for the distortion of the apparatus. Forced oscillation was imposed at $60 \mathrm{~Hz}$ with a $16 \mathrm{MPa}$ bias stress. Note that the bias stress and any apparent creep have been removed from these data. 
In reference to Figure 4, the second method of calculating attenuation utilizes the intersection of the Lissajous loop with the strain axis, $A$, and the strain magnitude at the maximum stress, C,

$$
Q^{-1}=\frac{A}{C}
$$

Finally, the attenuation can be calculated using the ratio of the imaginary component of the complex modulus, $E^{\prime \prime}$, to the real component of the complex modulus, $E^{\prime}$,

$$
Q^{-1}=\frac{E^{\prime \prime}}{E^{\prime}}
$$

where $E^{\prime \prime}$ and $E^{\prime}$ can be determined from the intersection of the Lissajous loop with the stress axis and the stress magnitude at the maximum strain, respectively.

Although these methods of calculating attenuation are theoretically equivalent, differences can arise associated with methods of data processing. The phase shift can be estimated by crosscorrelation methods or Fourier analysis. In contrast, estimates of attenuation based on the Lissajous loop can be made by hand from plots such as those in Figure 4, by taking the mean of $A, C, E^{\prime \prime}$, and $E^{\prime}$ for each loop, or by finding a best fit ellipse. We have compared all of these methods using the data described in the next section and find that calculated values of attenuation are reasonably insensitive to the method used, only differing by $25 \%$ at a maximum. Fourier analysis to find the phase shift has been chosen in analyzing the attenuation data presented below.

\subsection{Complex compliance of the apparatus}

In the apparatus configuration described above, displacement of the sample is inferred from measurements of the piston displacement referenced to the loading frame (i.e., with the linear encoder). Therefore, we apply a compliance correction to remove the distortion of the apparatus from the total measured distortion. To estimate the complex compliance of the apparatus, we conducted several forced-oscillation tests on single-crystal sapphire (an example is presented in Figure 4).

Although this apparatus is designed for investigating viscoelasticity at high temperatures, we only conduct calibration experiments at room temperature. This decision is based on the critical 
influence of the piston-sample contacts. The initial contact is inherently rough, which adds an additional compliance to the system due to deformation of asperities. For this reason, we use superglue (as described above) to provide a rigid contact between the sample and pistons. This approach clearly is not available at high temperatures. For tests at high temperature on materials of interest (rather than materials for calibration), an initial stage of creep at the beginning of the experiment allows asperities to flow and good sample-piston contact to be achieved. However, this approach is also not available for calibration runs at high temperatures because of the sapphire's strong resistance to creep (Firestone and Heuer, 1976). We emphasize that only the $\mathrm{SiC}$ pistons reach elevated temperatures, and therefore we only expect temperature to affect the apparatus compliance through the change in modulus of SiC. The Young's modulus of $\mathrm{SiC}$ is only reduced by $\sim 5 \%$ by increasing from room temperature to $1530^{\circ} \mathrm{C}$ (Fukuhara and Abe, 1993). Therefore, even if all of the apparatus compliance results from the $\mathrm{SiC}$ pistons and all of their length is brought to the same elevated temperature (the hot zone of the furnace is actually only $\sim 8 \mathrm{~cm}$ in length), we expect less than a $5 \%$ increase in the measured apparatus compliance.

To measure the apparatus compliance, we follow the work of Takei et al. (2011) and treat the apparatus and sample as a two-spring system connected in mechanical series (Takei et al., 2011). Takei et al. (2011) defined three complex spring compliances, given by

$$
\begin{gathered}
C_{\text {observed }}^{*}=\left(\frac{L}{S}\right) J_{\text {observed }}^{*}, \\
C_{\text {sample }}^{*}=\left(\frac{L}{S}\right) J_{\text {sample }}^{*} \\
C_{\text {apparatus }}^{*}=C_{\text {observed }}^{*}-C_{\text {sample }}^{*}
\end{gathered}
$$

where $C$ is a spring compliance (units of length/force), $L$ is the sample length, $S$ is the crosssectional area of the sample, $J$ is the compliance (units of inverse stress), “*” indicates a complex variable, and the subscripts indicate the component of the system with which the value is associated.

For our calibrations, $C^{*}$ sample corresponds to the known value for sapphire (Pezzotti et al., 1997; Wachtman and Lam, 1959), and $C^{*}$ observed corresponds to the value measured in the experiment. 
The difference between $C^{*}$ observed and $C^{*}$ sample, according to equation 8 , yields the complex spring compliance of the apparatus.

Calibration experiments were conducted over ranges of frequencies, load amplitudes, bias stresses, and sample dimensions, with multiple repeat tests at each set of conditions. Figure 5 presents the apparatus spring compliance as a function of frequency and load amplitude. Over the range of conditions tested, the real part of the apparatus compliance is on the order of 4 $\mathrm{nm} / \mathrm{N}$ with relatively little dependence on frequency. The imaginary part of the apparatus compliance is generally $<1 \mathrm{~nm} / \mathrm{N}$ and exhibits a subtle, negative frequency dependence at low frequencies. Only the imaginary part of the apparatus compliance exhibits a dependence on the load amplitude, with high amplitudes associated with increased compliance, primarily at intermediate frequencies.
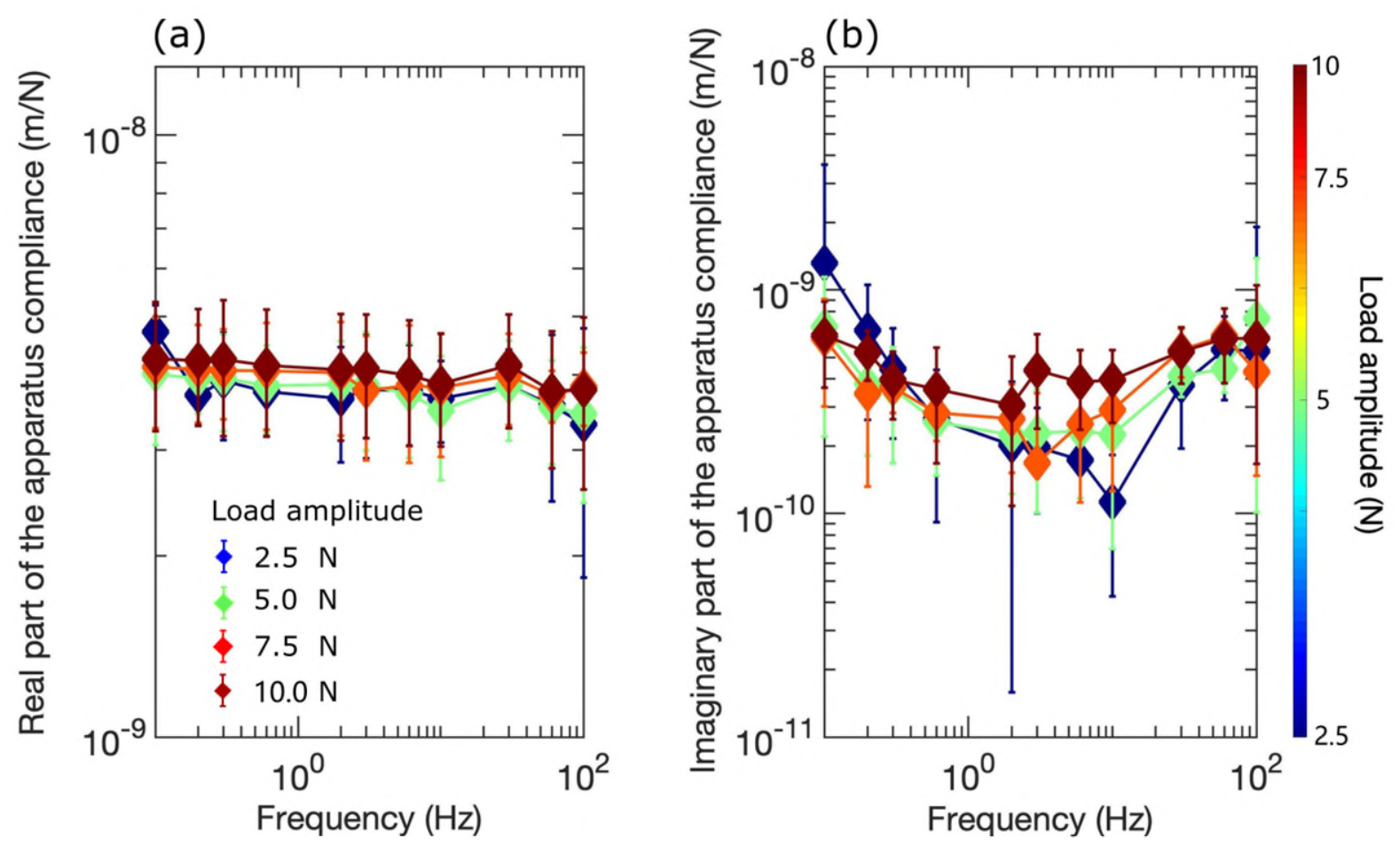

Figure 5: $C^{*}$ apparatus measured as a function of frequency and load amplitude at room-temperature using single-crystal sapphire as the calibration standard. (a) The real part of the apparatus compliance. (b) The imaginary part of the apparatus compliance. Multiple measurements are made at each value of frequency and amplitude. The symbol indicates the mean value, and the error bars denote one standard deviation.

We also examined the dependence of the apparatus compliance on the magnitude of the bias stress. As illustrated in Figure 6, we did not observe a systematic dependence of either the real or imaginary parts of the compliance on the bias stress. 

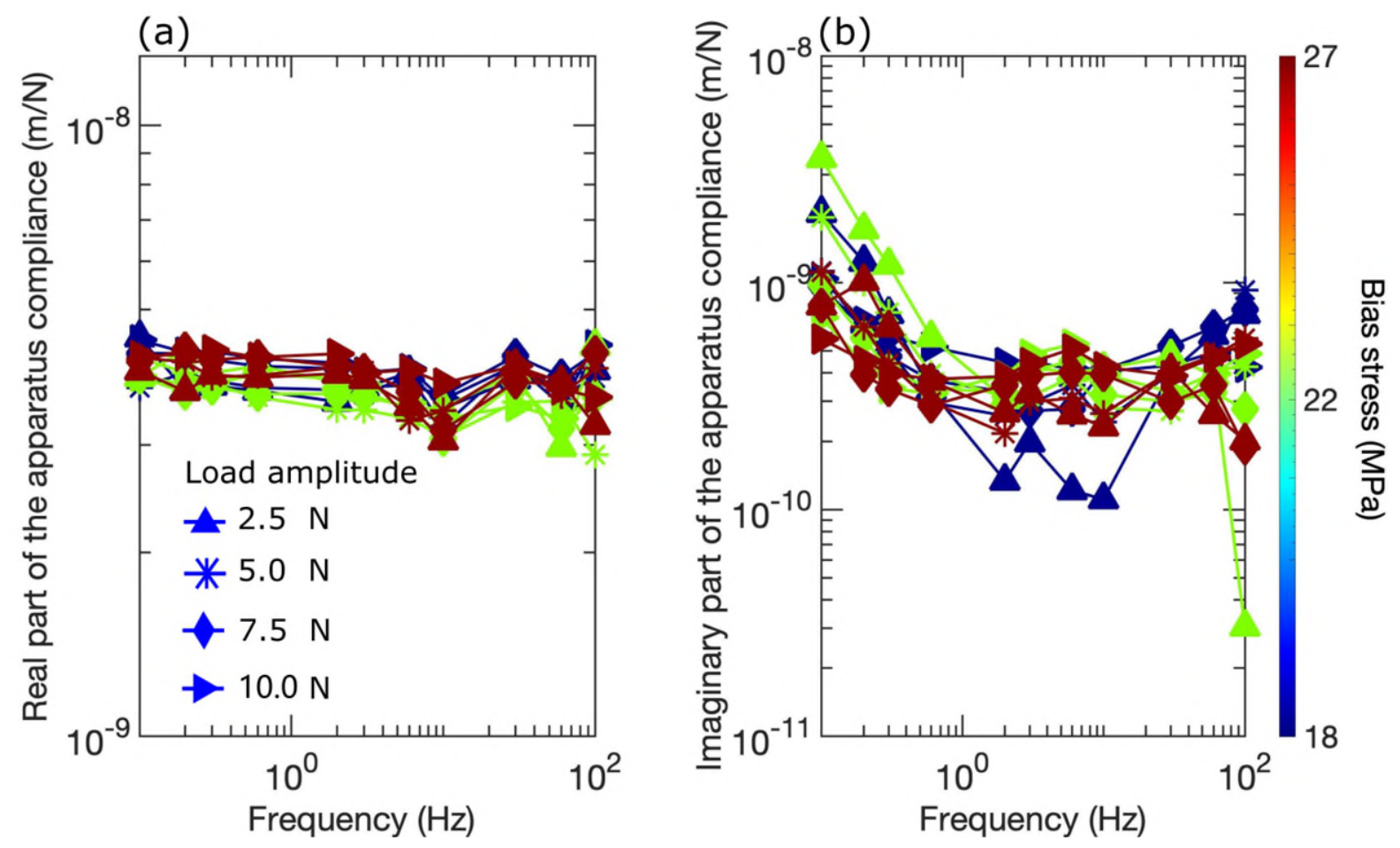

Figure 6: $C *$ apparatus measured as a function of frequency and bias stress at room-temperature using single-crystal sapphire as the calibration standard, with a fixed sample length. (a) The real part of the apparatus compliance. (b) The imaginary part of the apparatus compliance. Both (a) and (b) demonstrate that the compliance of the apparatus is essentially independent of the bias stress.

Finally, we examined the dependence of the apparatus compliance on the sample length. As illustrated in Figure 7, the real part of the apparatus compliance exhibits a systematic dependence on the sample length, with greater compliance as the sample length is decreased. We interpret this dependence to reflect the increase in length of the effective loading column as the lower piston is raised by the worm gear to accommodate shorter samples. The lack of a dependence of the imaginary component of the compliance on the sample length suggests that components other than the pistons are likely responsible for any relaxation. A likely source of the dissipation is the linear bearings for the guide rails on the lower piston. 

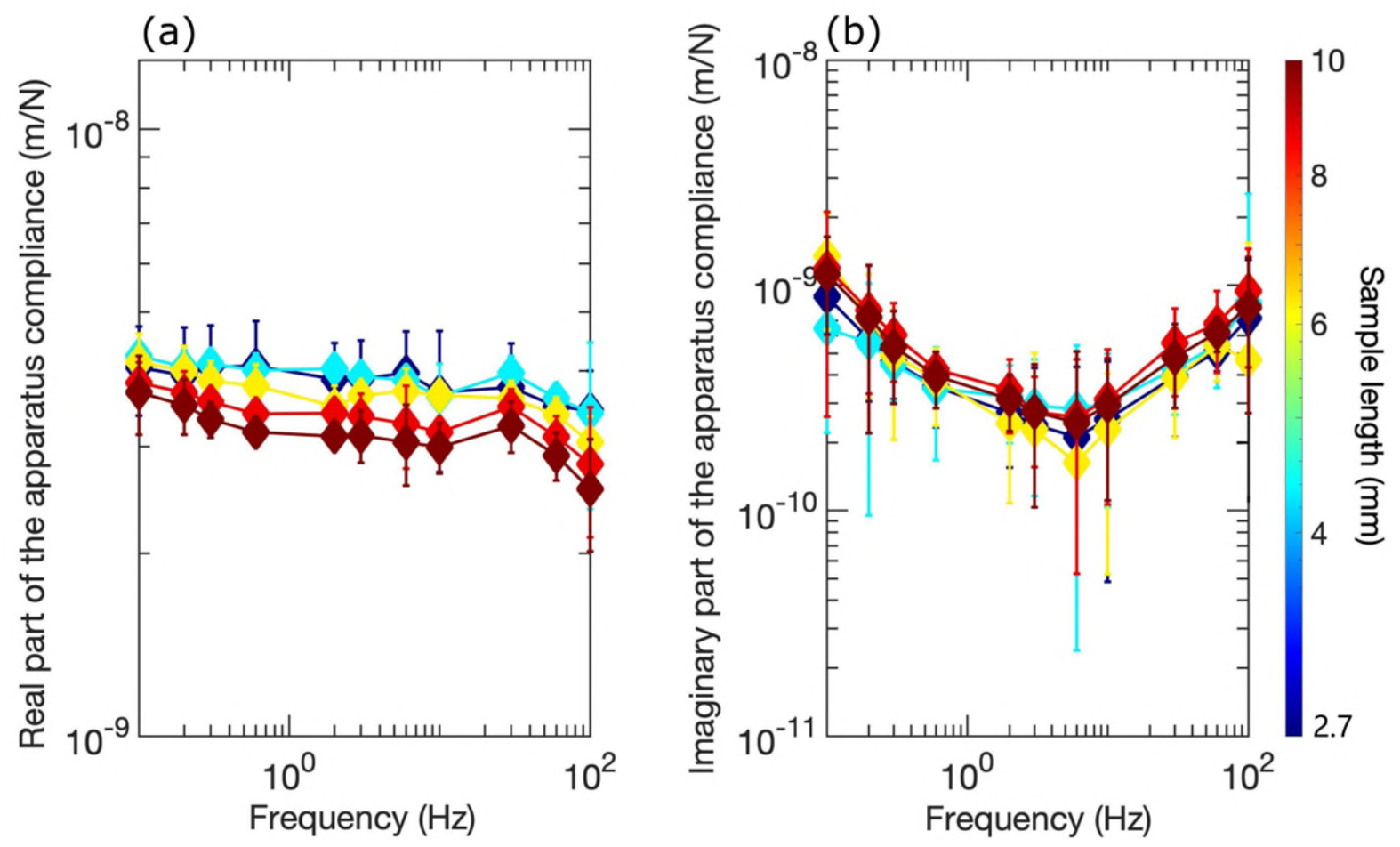

Figure 7: $C *$ apparatus measured as a function of frequency at room-temperature using single-crystal sapphire with different sample lengths as the calibration standard. (a) The real part of the apparatus compliance. (b) The imaginary part of the apparatus compliance. Multiple measurements are made at each value of frequency and sample length. The symbols indicate the mean values, and the error bars denote two standard deviations.

To define a best-fit apparatus compliance, we fit the data from these calibration experiments on sapphire to individual functions for the real and imaginary components. As demonstrated in Figures 5, 6, and 7, the real component of the compliance is dependent on the frequency and sample length, whereas the imaginary component of the apparatus compliance is primarily dependent on only the frequency. The functions fit to the real and imaginary components of the compliance are therefore constrained to only depend on those quantities. Fitting functions are determined by taking the mean of the compliances at a given set of independent variables and then conducting a linear interpolation among those means. Figure $8 \mathrm{a}$ presents the interpolated surface to the real component of the compliance and illustrates the tradeoff between frequency and sample length. Figure $8 \mathrm{~b}$ illustrates the interpolated curve to the imaginary component of the compliance. 
(a)

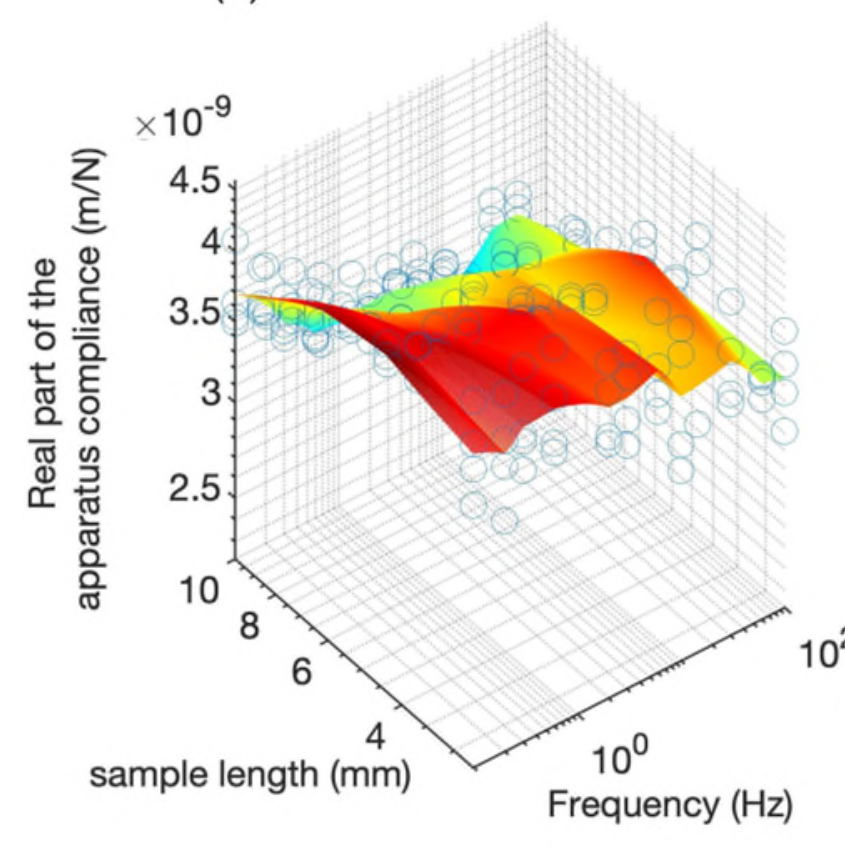

(b)

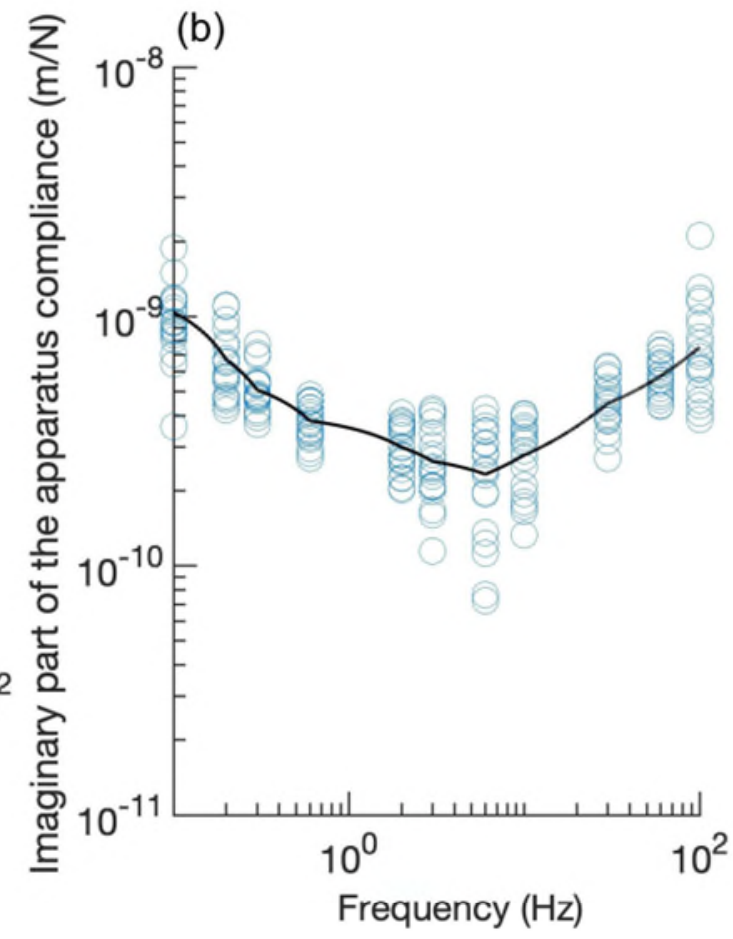

Figure 8: (a) Interpolated surface describing the dependence of the real part of the apparatus compliance on sample length and frequency. (b) Interpolated curve describing the dependence of the imaginary component of the apparatus compliance on frequency.

\subsection{Example measurements on stainless steel, aluminum, and acrylic}

To evaluate the ability of the apparatus and data processing method to estimate the attenuation, $Q^{-1}$, and Young's modulus, $E$, of a given sample, we made a variety of measurements on materials with known values of these quantities. We first calculated $Q^{-1}$ and $E$ using equations 1,2 , and 3 . We then use the relations

$$
\begin{gathered}
E=\left(J^{2}+J^{\prime \prime 2}\right)^{-\frac{1}{2}}, \\
Q^{-1}=\frac{J^{\prime \prime}}{J^{\prime}},
\end{gathered}
$$

to find $J^{\prime}$ and $J^{\prime \prime}$, which are the real and imaginary components of the complex compliance, respectively. With our measured values of the apparatus compliance, $C^{*}$ apparatus, we correct these observed spring compliances according to 


$$
J_{\text {sampale }}^{*}=J_{\text {observed }}^{*}-\frac{S}{L} C_{\text {apparatus }}^{*}
$$

We use this method to estimate $E$ and $Q^{-1}$ over a wide range of conditions for polycrystalline stainless steel, polycrystalline aluminum, and acrylic, as depicted in Figure 9.
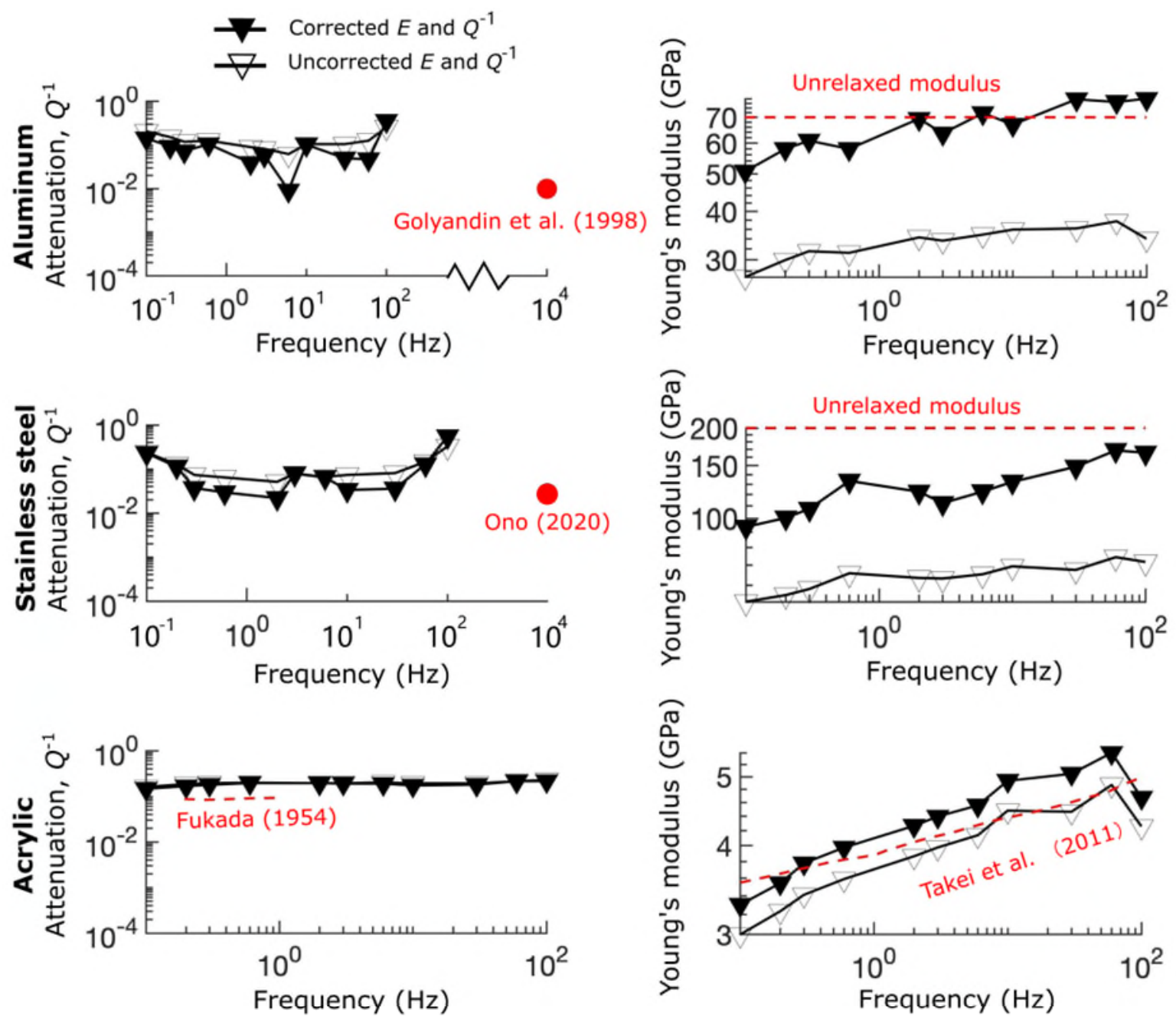

Figure 9: Attenuation, $Q^{-1}$, and Young's modulus measured as a function of frequency using aluminum, stainless steel, and acrylic at room temperature. The open and solid symbols indicate the attenuation and modulus before and after correction for the apparatus compliance, respectively. Red circles and dashed lines indicate reference values from other publications. Reference values of attenuation represent previous experiments conducted at similar stress amplitudes to our experiments. Unrelaxed moduli are given for aluminum and stainless steel, and the relaxed modulus (Takei et al., 2011) is given for acrylic. Forced oscillations were imposed at $5 \mathrm{~N}$ load amplitude for aluminum, stainless steel, and acrylic. The bias stress for aluminum and acrylic was $2.5 \mathrm{MPa}$, and the bias stress for stainless steel was $5 \mathrm{MPa}$. 
The attenuation and Young's modulus that we measured before and after compliance correction are presented in Figure 9. Comparisons have been made between the relaxed moduli measured in this study and the previously published, unrelaxed moduli of aluminum and stainless steel from Betekhtin et al. (2006) and Zener, (1948), respectively. At higher frequencies $(>30 \mathrm{~Hz})$, we reasonably recover the unrelaxed moduli of aluminum and stainless steel, demonstrating the feasibility of the compliance correction method. The corrected attenuation for stainless steel, aluminum, and acrylic is slightly smaller than that of the uncorrected attenuation data since the compliance correction procedure has removed the apparatus distortion from the measured distortion.

We also compare the attenuation of aluminum, stainless steel, and acrylic with previously published data from Golyandin et al. (1998), Ono (2020), and Fukada, (1954), respectively. Ono (2020) and Golyandin et al. (1998) conducted their forced-oscillation experiments at zero bias stress on annealed polycrystalline aluminum and annealed stainless steel, respectively, such that the initial dislocation densities in their samples were lower than in our own measurements. Therefore, it is not surprising that our attenuation measurements of aluminum and stainless steel are higher than those of Ono (2020) and Golyandin et al. (1998). However, the $Q^{-1}$ of acrylic exhibits a reasonable agreement with the measurement from Fukada (1954) since dislocations are not responsible for damping in this material. The corrected Young's modulus of acrylic is consistent with the data measured by Takei et al. (2011), even though the stress amplitudes employed here are much larger, which likely results from the attenuation of acrylic being much more sensitive to the stress amplitude than the reduction in modulus (Povolo and Goyanes, 1994).

\section{Discussion}

\subsection{Limits to measurement of attenuation and modulus}

With a quantitative understanding of the complex compliance of this apparatus, we can place bounds on the magnitudes of sample compliances that are reasonably measurable. Takei et al. (2011) suggested that accurate measurements of the viscoelastic properties of a material require displacements associated with distortion of the apparatus to be smaller than the displacements associated with distortion of the sample, that is, $C_{\text {sample }}^{\prime}>C_{\text {apparatus }}^{\prime}$ and $C^{\prime \prime}$ sample $>$ $C^{\prime \prime}$ apparatus. We modify these criteria by suggesting that the apparatus spring compliance can be larger than the sample spring compliance as long as the apparatus compliance is precisely 
known and suitably reproducible. Therefore, we suggest that the spring compliance of the sample must be larger than that of the error in the apparatus compliance, $C^{*}$ app,error, that is, $C_{\text {sample }}^{\prime}>C_{\text {app,error }}^{\prime}$ and $C^{\prime \prime}$ sample $>C^{\prime \prime}$ app,error. We define $C^{*}$ app,error as one standard deviation of the measurements of $C^{*}$ apparatus at a given set of conditions. We can therefore calculate the critical sample Young's modulus, $E_{\mathrm{c}}$, and the critical sample attenuation, $Q^{-1}$, which act as a maximum and minimum value, respectively, that can be reliably measured with this apparatus. These values are calculated from $C^{*}$ app,error by taking the sample dimensions into account

$$
\begin{aligned}
Q_{\mathrm{c}}^{-1} & =E \frac{S}{L} C_{\text {app,error }}^{\prime \prime}, \\
E_{\mathrm{c}} & =\frac{L}{S C_{\mathrm{app}, \mathrm{error}}^{\prime}} .
\end{aligned}
$$

An additional constraint arises from the measured timing error between the load and displacement signals (Figure 2). The horizontal, dash-dot lines in Figure 10 indicate a minimum measurable value of $Q^{-1}$, above which the predicted time delay between the load and displacement signals, $\Delta t$, is greater than that caused by a timing error of $200 \mu$ s. These minima in $Q^{-1}$ vary linearly with the oscillation frequency, with an increase in frequency from $10 \mathrm{~Hz}$ to $30 \mathrm{~Hz}$ leading to a factor of 3 increase in the minimum measurable attenuation. Although this additional constraint limits the maximum frequencies we can work with, we note that most previous apparatus designed for working at high homologous temperatures in geological materials are limited to much lower frequencies $(<10 \mathrm{~Hz})$. 


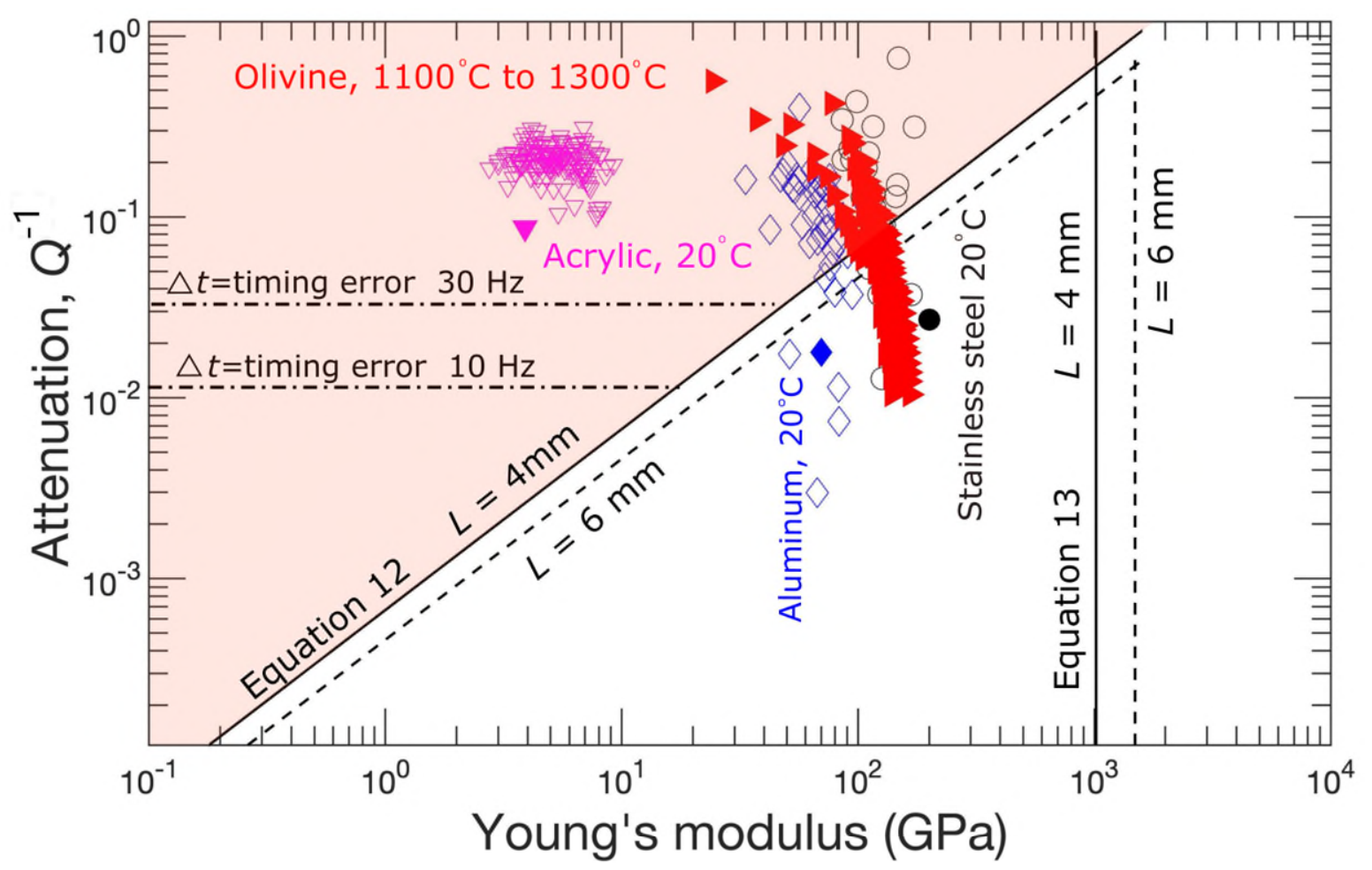

Figure 10: Bounds on reliable measurements of attenuation and Young's modulus by the criterion suggested in this study (equations 12 and 13). The shaded area indicates the values of the sample attenuation and sample modulus that result in sample displacements larger than the error in the apparatus displacement. These bounds are dependent on the sample length, $L$. Also presented are example values of $E$ and $Q^{-1}$ for other materials. Filled markers refer to previously published values, and open markers refer to values measured in this study. The horizontal dashdot lines indicate a minimum value of $Q^{-1}$, above which the predicted time delay associated with dissipation is greater than a timing error of $200 \mu \mathrm{s}$, which is dependent on the oscillation frequency. Data for acrylic are from Yee and Takemori (1982), data for aluminum are from Lakes (2009), and data for stainless steel are from Ono (2020). Data for olivine are from Jackson et al. (2010), Faul and Jackson (2015) and Cline et al. (2018).

Also depicted in Figure 10 are the values for $E$ and $Q^{-1}$ of aluminum (open black diamond) and stainless steel (open blue circle) measured in this study along with previously published data for olivine (solid red right triangles), aluminum (solid blue diamonds), and stainless steel (solid black circles). According to equation 13, all of these materials should have Young's moduli within the measurable range, which is confirmed by our analysis in Figure 9. Similarly, the attenuation of acrylic is well within the measurable range. In contrast, the published values of attenuation for aluminum and steel are below our threshold for accurate measurement. The measurements made here for attenuation of these materials sit close to the threshold defined 
by equation 12. As described above, the discrepancy between our measurements and previously published data may arise from the increased dislocation densities likely in our samples. However, our sensitivity analysis discussed in this section additionally suggests that the measured values of attenuation may be too high because we simply do not have the ability to resolve lower values.

As an example of applicability to geological materials, Figure 10 also compares our constraints to previous measurements of attenuation in olivine. The Young's modulus of olivine is clearly accessible with this apparatus, although only about half of the previously published olivine data (solid red triangles) lies within the range of measurable values of attenuation. We suggest that measurements of attenuation will be most successful if conditions are chosen to maximize the dissipation of the sample, such as at high temperatures or high stress amplitudes.

\subsection{Comparison to other apparatus}

A variety of apparatus have been developed to measure the attenuation of geological materials at elevated temperatures, with either forced oscillation Gribb and Copper (1998); Jackson and Paterson (1993); Sundberg and Cooper (2010) or in a resonant torsion pendulum (Gueguen et al., 1989). These apparatus were primarily designed to investigate attenuation in the linear regime for application to seismic-wave attenuation. However, these apparatus designs are not able to apply a bias stress, which means they are unable to measure attenuation while the material is simultaneously creeping. Thus, to investigate the effect of defects related to creep (e.g., dislocations) on attenuation, samples must be predeformed in a separate experiment prior to measurement of attenuation. For example, to investigate the role of dislocation density on attenuation in olivine, Gueguen et al. (1989) measured the attenuation of $\mathrm{Fo}_{100}$ (100\% forsterite) predeformed in a 1-atm creep apparatus at $20 \mathrm{MPa}$ differential stress, and Farla et al. (2012) measured the attenuation of predeformed aggregates of Fo90 (iron-bearing forsterite) at 230 $\mathrm{MPa}$ differential stress. A downside of this approach is that, during subsequent viscoelastic experiments at high temperatures, recovery acts to modify the specific arrangement of dislocations, the dislocation density, and the internal stress distribution as the attenuation is measured.

There are several existing apparatus designed for geological applications that do allow a high bias stress to be applied during oscillatory experiments, although they are limited to low temperatures. Takei et al. (2011) describe an apparatus that allows creep and attenuation to be 
measured simultaneously at temperatures up to $50^{\circ} \mathrm{C}$. This apparatus has been specifically used for characterizing organic, crystalline materials that act as rock analogues. McCarthy et al. (2016) described viscoelastic measurements with a high bias stress utilizing a servomechanical apparatus designed for cryogenic conditions, and their experiments on ice revealed nonlinear behavior associated with dislocation activity.

The apparatus that we introduce here provides new and key capabilities for measuring the viscoelasticity of geological materials. This apparatus is capable of conducting forcedoscillation tests in tandem with an appreciable bias stress (maximum loads of $5 \mathrm{kN}$ ) while at temperatures up to $1330^{\circ} \mathrm{C}$ in a controlled chemical environment. We are therefore able to explore amplitude-dependent, nonlinear viscoelasticity at high homologous temperatures in minerals with high melting temperatures. The ability to apply a bias stress allows the viscoelastic properties to be investigated during creep, including as the microstructure evolves during the initial increments of transient creep. Furthermore, we can potentially apply a lowfrequency oscillation to the bias stress, which has been an extremely valuable technique for investigating dislocation dynamics in metals at low temperatures (e.g., Gremaud and Bujard, 1985).

\section{Conclusions}

A high-temperature, uniaxial creep apparatus has been developed in this study to investigate the nonlinear attenuation of crystalline materials at a wide range of temperatures $\left(25-1300{ }^{\circ} \mathrm{C}\right)$, using forced-oscillations. This apparatus is primarily designed for applying oscillatory compressional stress to estimate attenuation and Young's modulus. This apparatus allows us to apply a high bias stress in addition to forced oscillations, enabling us to investigate the effect of creep-derived defects (e.g., dislocations) on attenuation and modulus.

We performed a variety of calibration tests, including quantifying the timing errors in the data acquisition system, the resonant frequencies of the apparatus, and the complex compliance of the apparatus. Based on the results from the calibration tests conducted on single-crystal sapphire, the real part of the apparatus compliance is on the order of $4 \mathrm{~nm} / \mathrm{N}$ with relatively little dependence on frequency. The imaginary part of the apparatus compliance is generally $<1 \mathrm{~nm} / \mathrm{N}$, illustrating a subtle negative frequency dependence at low frequencies. The real part of the apparatus compliance exhibits a systematic dependence on the sample length, with greater compliances as the sample length is decreased. There is no systematic dependence of 
either the real or imaginary parts of the compliance on the bias stress. We define best-fit functions to allow the real and imaginary component of the compliance to be estimated at any frequency, amplitude, or sample length within the ranges explored here.

With subsequent measurements of the compliance of aluminum, steel, and acrylic, we demonstrate that correction of data for the apparatus compliance yields reasonable measurements of $Q^{-1}$ and $E$. We have constrained the upper limit of $E$ and the lower limit of $Q^{-1}$ that can be accurately measured.

\section{Acknowledgements}

The authors wish to acknowledge the considerable effort from Physik Instrumente in the design and construction of this apparatus, including Geraint Green, John Hopkins, and Huw Prosser. Invaluable design and construction input was also provided by Jamie Long and James King in the Earth Sciences Workshop at the University of Oxford. RC acknowledges support from the Great Britain-China Educational Trust, Mineralogical Society of Great Britain \& Ireland, and the Linacre Travel Fund. LH and CT acknowledge support from the Natural Environment Research Council, grant 1710DG008/JC4. LH and DW acknowledge support from the Natural Environment Research Council, grant NE/M000966/1. DW acknowledges support from the Netherlands Organisation for Scientific Research, User Support Programme Space Research, grant ALWGO.2018.038. LH recognizes funds used to develop the apparatus from the John Fell Fund at the University of Oxford.

\section{Data availability}

The data that support the findings of this study are available from the corresponding author upon reasonable request.

\section{Supplementary material}

\section{Supplement A: All measurements of apparatus compliance used in this study}

\begin{tabular}{|c|c|c|c|c|c|c|}
\hline $\begin{array}{l}\text { Real part of } \\
\text { apparatus } \\
\text { compliance } \\
(\mathrm{nm} / \mathrm{N})\end{array}$ & $\begin{array}{l}\text { Imaginary part } \\
\text { of apparatus } \\
\text { compliance } \\
(\mathrm{nm} / \mathrm{N})\end{array}$ & $\begin{array}{l}\text { Frequency } \\
(\mathrm{Hz})\end{array}$ & $\begin{array}{l}\text { Load amplitude } \\
\text { (N; peak-to- } \\
\text { peak) }\end{array}$ & $\begin{array}{l}\text { Temperature } \\
\left({ }^{\circ} \mathrm{C}\right)\end{array}$ & $\begin{array}{l}\text { Sample length } \\
(\mathrm{mm})\end{array}$ & $\begin{array}{l}\text { Cross- } \\
\text { sectional } \\
\text { area }\left(\mathrm{mm}^{-2}\right)\end{array}$ \\
\hline $\begin{array}{l}5.11 \\
3.43 \\
3.30 \\
3.27\end{array}$ & $\begin{array}{l}0.60 \\
0.69 \\
0.11 \\
0.09\end{array}$ & $\begin{array}{l}0.1 \\
0.2 \\
0.3 \\
0.6\end{array}$ & 2.5 & 25 & 2.71 & 12.6 \\
\hline
\end{tabular}




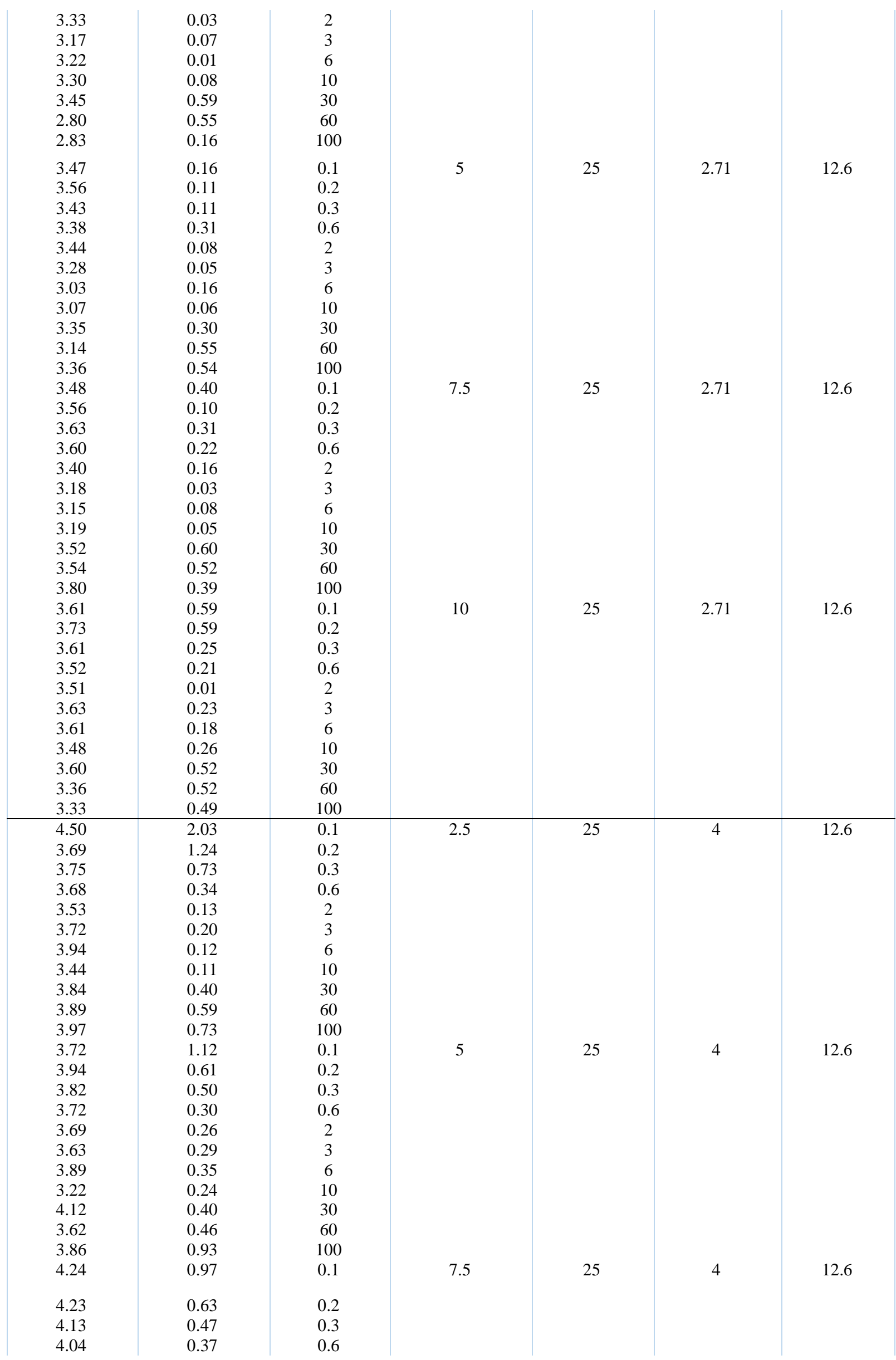




\begin{tabular}{|c|c|c|c|c|c|c|}
\hline $\begin{array}{l}4.00 \\
3.73 \\
3.75 \\
3.61 \\
4.20 \\
3.88 \\
4.24 \\
4.46 \\
4.25 \\
4.27 \\
4.15 \\
4.09 \\
3.94 \\
4.00 \\
3.56 \\
4.05 \\
3.49 \\
4.38\end{array}$ & $\begin{array}{l}0.33 \\
0.27 \\
0.28 \\
0.41 \\
0.53 \\
0.64 \\
0.76 \\
1.04 \\
0.67 \\
0.58 \\
0.52 \\
0.44 \\
0.45 \\
0.41 \\
0.41 \\
0.43 \\
0.49 \\
0.42\end{array}$ & $\begin{array}{c}2 \\
3 \\
6 \\
10 \\
30 \\
60 \\
100 \\
0.1 \\
0.2 \\
0.3 \\
0.6 \\
2 \\
3 \\
6 \\
10 \\
30 \\
60 \\
100\end{array}$ & 10 & 25 & 4 & 12.6 \\
\hline $\begin{array}{l}4.56 \\
3.60 \\
3.99 \\
3.87 \\
3.28 \\
3.64 \\
4.15 \\
3.77 \\
3.79 \\
3.84 \\
3.83 \\
2.98 \\
4.09 \\
4.22 \\
4.16 \\
4.07 \\
3.89 \\
4.25 \\
3.78 \\
4.11 \\
4.22 \\
3.86 \\
3.90 \\
4.45 \\
4.32 \\
4.42 \\
4.42 \\
4.41 \\
4.15 \\
4.50 \\
4.40 \\
4.41 \\
3.88 \\
3.61 \\
4.64 \\
4.82 \\
4.74 \\
4.69 \\
4.63 \\
4.57 \\
4.41 \\
4.22 \\
4.71 \\
4.38 \\
4.24\end{array}$ & $\begin{array}{l}4.13 \\
0.22 \\
0.55 \\
0.55 \\
0.25 \\
0.27 \\
0.17 \\
0.13 \\
0.04 \\
0.63 \\
0.48 \\
0.75 \\
0.89 \\
0.85 \\
0.40 \\
0.31 \\
0.33 \\
0.26 \\
0.25 \\
0.18 \\
0.50 \\
0.43 \\
0.44 \\
1.65 \\
0.77 \\
0.43 \\
0.43 \\
0.35 \\
0.32 \\
0.05 \\
0.32 \\
0.67 \\
0.64 \\
0.76 \\
0.38 \\
0.66 \\
0.59 \\
0.47 \\
0.61 \\
0.45 \\
0.68 \\
0.46 \\
0.58 \\
0.67 \\
1.00\end{array}$ & $\begin{array}{c}0.1 \\
0.2 \\
0.3 \\
0.6 \\
2 \\
3 \\
6 \\
10 \\
20 \\
30 \\
60 \\
100 \\
0.1 \\
0.2 \\
0.3 \\
0.6 \\
2 \\
3 \\
6 \\
10 \\
30 \\
60 \\
100 \\
0.1 \\
0.2 \\
0.3 \\
0.6 \\
2 \\
3 \\
6 \\
10 \\
30 \\
60 \\
100 \\
0.1 \\
0.2 \\
0.3 \\
0.6 \\
2 \\
3 \\
6 \\
10 \\
30 \\
60 \\
100\end{array}$ & 2.5 & 25 & 6 & 12.6 \\
\hline $\begin{array}{l}4.24 \\
8.53 \\
7.47 \\
7.03 \\
6.65\end{array}$ & $\begin{array}{l}1.00 \\
1.19 \\
0.66 \\
0.59 \\
0.47\end{array}$ & $\begin{array}{l}100 \\
0.1 \\
0.2 \\
0.3 \\
0.6\end{array}$ & 2.5 & 25 & 8 & 12.6 \\
\hline
\end{tabular}




\begin{tabular}{|c|c|c|c|c|c|c|}
\hline & & & \multirow[b]{9}{*}{5} & \multirow{9}{*}{25} & \multirow[b]{9}{*}{8} & \multirow[b]{9}{*}{12.6} \\
\hline 6.45 & 0.61 & 2 & & & & \\
\hline 6.24 & 0.45 & 3 & & & & \\
\hline 5.94 & 0.68 & 6 & & & & \\
\hline 5.31 & 0.46 & 10 & & & & \\
\hline 6.07 & 0.58 & 30 & & & & \\
\hline 5.31 & 0.67 & 60 & & & & \\
\hline 4.79 & 1.00 & 100 & & & & \\
\hline 6.92 & 1.19 & 0.1 & & & & \\
\hline 6.67 & 3.12 & 0.2 & & & & \\
\hline 6.46 & 1.80 & 0.3 & & & & \\
\hline 6.41 & 0.83 & 0.6 & & & & \\
\hline 6.19 & 0.49 & 2 & & & & \\
\hline 5.65 & 0.51 & 3 & & & & \\
\hline 5.42 & 0.30 & 6 & & & & \\
\hline 5.67 & 0.58 & 10 & & & & \\
\hline 6.04 & 0.58 & 30 & & & & \\
\hline 5.60 & 0.73 & 60 & & & & \\
\hline 5.57 & 1.11 & 100 & & & & \\
\hline 7.64 & 0.88 & 0.1 & 7.5 & 25 & 8 & 12.6 \\
\hline 7.37 & 1.30 & 0.2 & & & & \\
\hline 7.32 & 0.77 & 0.3 & & & & \\
\hline 7.16 & 0.97 & 0.6 & & & & \\
\hline 7.07 & 0.58 & 2 & & & & \\
\hline 6.93 & 0.55 & 3 & & & & \\
\hline 6.71 & 0.52 & 6 & & & & \\
\hline 6.42 & 0.46 & 10 & & & & \\
\hline 6.62 & 0.49 & 30 & & & & \\
\hline 6.03 & 0.64 & 60 & & & & \\
\hline 5.99 & 0.90 & 100 & & & & \\
\hline 7.01 & 0.82 & 0.1 & 10 & 25 & 8 & 12.6 \\
\hline 6.87 & 1.12 & 0.2 & & & & \\
\hline 7.13 & 0.59 & 0.3 & & & & \\
\hline 6.89 & 0.63 & 0.6 & & & & \\
\hline 6.77 & 0.61 & 2 & & & & \\
\hline 6.72 & 0.83 & 3 & & & & \\
\hline 6.57 & 0.90 & 6 & & & & \\
\hline 6.46 & 0.79 & 10 & & & & \\
\hline 6.71 & 0.96 & 30 & & & & \\
\hline 6.11 & 0.91 & 60 & & & & \\
\hline 5.91 & 1.17 & 100 & & & & \\
\hline
\end{tabular}

\section{References}

Abers, G.A., Fischer, K.M., Hirth, G., 2014. Reconciling mantle attenuation-temperature relationships from seismology, petrology, and laboratory measurements. Geochem. Explor. Environ. Analy. Agata, R., Barbot, S.D., Fujita, K., Hyodo, M., Iinuma, T., Nakata, R., Ichimura, T., Hori, T., 2019. Rapid mantle flow with power-law creep explains deformation after the 2011 Tohoku megaquake. Nat. Commun. 10, 1385.

Bai, Q., Kohlstedt, D.L., 1992. High-temperature creep of olivine single crystals III. Mechanical 
results for unbuffered samples and creep mechanisms. Philos. Mag. A 66, 1149-1181.

Betekhtin, V.I., Kadomtsev, A.G., Kardashev, B.K., 2006. Elasticity and anelasticity of microcrystalline aluminum samples having various deformation and thermal histories. Phys. Solid State 48, 1506-1512.

Cheng, T.S., Davies, A., Summerfield, A., Cho, Y., Cebula, I., Hill, R.J.A., Mellor, C.J., Khlobystov, A.N., Taniguchi, T., Watanabe, K., Beton, P.H., Foxon, C.T., Eaves, L., Novikov, S.V., 2016. High temperature MBE of graphene on sapphire and hexagonal boron nitride flakes on sapphire. J. Vac. Sci. Technol. B Microelectron. Nanometer Struct. Process. Meas. Phenom. 34, 02L101.

Cline, C.J., Ii, Faul, U.H., David, E.C., Berry, A.J., Jackson, I., 2018. Redox-influenced seismic properties of upper-mantle olivine. Nature 555, 355-358.

Farla, R.J.M., Jackson, I., Fitz Gerald, J.D., Faul, U.H., Zimmerman, M.E., 2012. Dislocation damping and anisotropic seismic wave attenuation in Earth's upper mantle. Science 336, 332335.

Faul, U., Jackson, I., 2015. Transient Creep and Strain Energy Dissipation: An Experimental Perspective. Annu. Rev. Earth Planet. Sci. 43, 541-569.

Firestone, R.F., Heuer, A.H., 1976. Creep deformation of 0 sapphire. J. Am. Ceram. Soc. 59, 24-29. Freed, A.M., Hirth, G., Behn, M.D., 2012. Using short-term postseismic displacements to infer the ambient deformation conditions of the upper mantle. J. Geophys. Res. [Solid Earth] 117.

Fukada, E., 1954. The Relation between Dynamic Elastic Modulus, Internal Friction, Creep and Stress Relaxation in Polymethyl Methacrylate. J. Phys. Soc. Jpn. 9, 786-789.

Fukuhara, M., Abe, Y., 1993. High-temperature elastic moduli and internal frictions of $\alpha$-SiC ceramic. J. Mater. Sci. Lett. 12, 681-683.

Golyandin, S.N., Sapozhnikov, K.V., Emel'yanov, Y.A., Sinani, A.B., Nikanorov, S.P., Kustov, S.B., Robinson, W.H., 1998. Influence of temperature and strain on the amplitude-dependent internal friction of high-purity aluminum. Phys. Solid State 40, 1667-1671.

Gremaud, G., Bidaux, J.E., Benoit, W., 1987. Etude a basse frequence des pics de frottement interieur associes a une transition de phase du 1er ordre. Helv. Phys. Acta 60, 947-958.

Gremaud, G., Bujard, M., 1985. RECENT PROGRESS IN DISLOCATION STUDIES USING BIAS 
STRESS EXPERIMENTS. Le Journal de Physique Colloques 46, C10-315-C10-320.

Gribb, T.T., Cooper, R.F., 1998. A high-temperature torsion apparatus for the high-resolution characterization of internal friction and creep in refractory metals and ceramics: Application to the seismic-frequency, dynamic response of Earth's upper mantle. Rev. Sci. Instrum. 69, 559564.

Gueguen, Y., Darot, M., Mazot, P., Woirgard, J., 1989. Q-1 of forsterite single crystals. Phys. Earth Planet. Inter. 55, 254-258.

Jackson, I., Faul, U.H., 2010. Grain size-sensitive viscoelastic relaxation in olivine: Towards a robust laboratory-based model for seismological application. Phys. Earth Planet. Inter. 183, 151-163.

Jackson, I., Paterson, M.S., 1993. A high-pressure, high-temperature apparatus for studies of seismic wave dispersion and attenuation. Pure Appl. Geophys. 141, 445-466.

Kaiser, G., Pechhold, W., 1969. Dynamic-mechanical investigations for the study of dislocation motion during plastic flow. Acta Metall. 17, 527-537.

Lakes, R.S., 2009. Viscoelastic Materials. Cambridge University Press.

Lau, H.C.P., Holtzman, B.K., 2019. “Measures of Dissipation in Viscoelastic Media” Extended: Toward Continuous Characterization Across Very Broad Geophysical Time Scales. Geophys. Res. Lett. 46, 9544-9553.

Li, L., Weidner, D.J., 2007. Energy dissipation of materials at high pressure and high temperature. Rev. Sci. Instrum. 78, 053902.

Masuti, S., Barbot, S.D., Karato, S.-I., Feng, L., Banerjee, P., 2016. Upper-mantle water stratification inferred from observations of the 2012 Indian Ocean earthquake. Nature 538, 373-377.

McCarthy, C., Cooper, R.F., 2016. Tidal dissipation in creeping ice and the thermal evolution of Europa. Earth Planet. Sci. Lett. 443, 185-194.

McCarthy, C., Takei, Y., Hiraga, T., 2011. Experimental study of attenuation and dispersion over a broad frequency range: 2. The universal scaling of polycrystalline materials. J. Geophys. Res. [Solid Earth] 116. https://doi.org/10.1029/2011JB008384

Moore, J.D.P., Yu, H., Tang, C.-H., Wang, T., Barbot, S., Peng, D., Masuti, S., Dauwels, J., Hsu, Y.J., Lambert, V., Nanjundiah, P., Wei, S., Lindsey, E., Feng, L., Shibazaki, B., 2017. Imaging the 
distribution of transient viscosity after the $2016 \mathrm{Mw}$ 7.1 Kumamoto earthquake. Science 356, $163-167$.

Ono, K., 2020. A Comprehensive Report on Ultrasonic Attenuation of Engineering Materials, Including Metals, Ceramics, Polymers, Fiber-Reinforced Composites, Wood, and Rocks. NATO Adv. Sci. Inst. Ser. E Appl. Sci. 10, 2230.

Pezzotti, G., Ota, K. 'ichi, Kleebe, H.-J., 1997. Viscous Slip along Grain Boundaries in ChlorineDoped Silicon Nitride. J. Am. Ceram. Soc. 80, 2341-2348.

Povolo, F., Goyanes, S.N., 1994. Amplitude-Dependent Dynamical Behavior of Poly(methyl methacrylate). Polym. J. 26, 1054-1062.

Qiu, Q., Moore, J.D.P., Barbot, S., Feng, L., Hill, E.M., 2018. Transient rheology of the Sumatran mantle wedge revealed by a decade of great earthquakes. Nat. Commun. 9, 995.

Sapozhnikov, K.V., Kustov, S.B., 1997. Effect of temperature on the amplitude dependences of the acoustoplastic effect and internal friction during deformation of crystals. Philos. Mag. A 76, $1153-1168$.

Sundberg, M., Cooper, R.F., 2010. A composite viscoelastic model for incorporating grain boundary sliding and transient diffusion creep; correlating creep and attenuation responses for materials with a fine grain size. Philos. Mag. 90, 2817-2840.

Takei, Y., Fujisawa, K., McCarthy, C., 2011. Experimental study of attenuation and dispersion over a broad frequency range: 1 . The apparatus. J. Geophys. Res. 116, 65.

Takei, Y., Karasawa, F., 2014. Temperature, grain size, and chemical controls on polycrystal anelasticity over a broad frequency range extending into the seismic range. J. Geophys. Res.

Twiss, R.J., 1986. Variable sensitivity piezometric equations for dislocation density and subgrain diameter and their relevance to olivine and quartz. Mineral and Rock Deformation: Laboratory Studies, AGU Geophysical Monographs 36, 247-263.

Wachtman, J.B., Jr., Lam, D.G., Jr., 1959. Young's Modulus of Various Refractory Materials as a Function of Temperature. J. Am. Ceram. Soc. 42, 254-260.

Wallis, D., Hansen, L.N., Kumamoto, K.M., Thom, C.A., 2020. Dislocation interactions during lowtemperature plasticity of olivine and their impact on the evolution of lithospheric strength. Earth 
Planet. Sci. Lett.

Wang, J., Guo, B., Zhao, Q., Zhang, C., Zhang, Q., Chen, H., Sun, J., 2017. Dependence of material removal on crystal orientation of sapphire under cross scratching. J. Eur. Ceram. Soc. 37, 24652472.

Yee, A.F., Takemori, M.T., 1982. Dynamic bulk and shear relaxation in glassy polymers. I.

Experimental techniques and results on PMMA. Journal of Polymer Science: Polymer Physics Edition 20, 205-224.

Zener, C., 1948. Elasticity and anelasticity of metals. University of Chicago press. 